\title{
Identification of candidate genes associated with tubal origin of high-grade serous ovarian cancer
}

\author{
LI XIANG $^{1}$, GUOHUA RONG $^{2}$, JING ZHAO $^{3}$, ZHENYAN WANG $^{1}$ and FENGFENG SHI ${ }^{4}$ \\ ${ }^{1}$ Department of Gynecology, The Affiliated Hospital of Qingdao University; ${ }^{2}$ Department of Breast Surgery, \\ Qingdao Municipal Hospital (Group); Departments of ${ }^{3}$ Pathology and ${ }^{4}$ Ultrasound, \\ The Affiliated Hospital of Qingdao University, Qingdao, Shandong 266000, P.R. China
}

Received February 13, 2017; Accepted January 23, 2018

DOI: $10.3892 / \mathrm{ol} .2018 .8346$

\begin{abstract}
Evidence indicates that high-grade serous ovarian carcinoma arises from the fallopian tube, rather than ovarian surface epithelium. This is termed the "tubal origin' theory. The aim of the present study was to compare the immunophenotype and gene expression profiling among high-grade serous ovarian carcinoma (HGSOC), fallopian tube epithelium (FTE) and ovarian surface epithelium (OSE) based on tubal origin theory, and identify the differential genes associated with ovarian carcinogenesis. A total of 61 cases of fresh tissue samples including 21 cases of HGSOC, 20 cases of OSE, and 20 cases of FTE were obtained following surgical resection. Immunostaining was performed to detect the expression of PAX8, which has been considered as a potential immunophenotype marker of Müllerian origin. Illumina BeadChip was applied for gene expression profiling. Reverse transcription-quantitative polymerase chain reaction (RT-qPCR) was performed to confirm the differential expression of candidate genes between HGSOC and FTE. The results of the present study demonstrated that PAX8 was highly expressed in HGSOC $(19 / 21,90.4 \%)$ and FTE $(20 / 20,100 \%)$, but not in OSE $(3 / 20,14.3 \%)$. A dendrogram generated by cluster analysis indicated a higher similarity of gene expression profile between HGSOC and FTE than OSE. A total of 2,412 differentially expressed genes were identified (absolute fold change $>2$ ) between HGSOC and FTE, including 822 upregulated genes in cancer and 1,590 downregulated genes. S100 calcium binding protein $\mathrm{P}$, Ras-interacting protein 1 , Wnt family member 5A, tumor-associated calcium signal transducer 2, Dickkopf Wnt signaling pathway inhibitor 3 and tumor suppressor candidate 3 genes were identified as candidate markers, of which the differential gene expression
\end{abstract}

Correspondence to: Dr Li Xiang, Department of Gynecology, The Affiliated Hospital of Qingdao University, 59 Haier Road, Qingdao, Shandong 266000, P.R. China

E-mail: ffprivate@163.com

Key words: high-grade serous ovarian carcinoma, tubal origin, gene expression profiling, candidate markers in HGSOC and FTE was confirmed by RT-qPCR $(\mathrm{P}<0.05)$. The results indicate the presence of a greater similarity in the immunophenotype and gene expression profile of HGSOC and FTE, when compared with OSE, which was consistent with the tubal origin theory of HGSOC.

\section{Introduction}

Ovarian cancer is the leading cause of mortality among women with gynecological malignancies. It is frequently diagnosed at an advanced, incurable stage and has a poor survival rate, owing to its asymptomatic development (1-3). High-grade serous ovarian cancer (HGSOC) is the most aggressive and common subtype of ovarian cancer, accounting for two-thirds of cancer-associated mortalities (1). HGSOC is characterized by a mutation in tumor protein p53 (TP53), a low rate of other mutations and extensive DNA copy number changes $(4,5)$. Therefore, it is crucial to elucidate the etiology and carcinogenesis of ovarian cancer, particularly in patients with HGSOC. The traditional theory, which states that ovarian cancer originates from ovarian surface epithelium (OSE), has been challenged fundamentally in recent years $(6,7)$. Currently, a new paradigm for the pathogenesis of HGSOCs, with its origins in the fallopian tube epithelium (FTE), has been proposed; it is supported by numerous studies and has been termed 'tubal origin' theory (8-10). It has been identified that paired box gene 8 (PAX8), which is considered as the marker of the organs of Müllerian origin including FTE, was highly expressed in HGSOC and FTE, but not OSE (11). PAX8 serves an important role as a distinguished factor in comparing the immunophenotype of HGSOC, FTE and OSE. It indicates that the immunophentype of HGSOC and FTE showed more similarity than that of OSE, which supports 'tubal origin' theory (11).

Considering the traditional theory focuses on the differentially expressed genes between HGSOC and OSE, it is of clinical importance to establish the true origin of HGSOC and identify the genes differentially expressed between HGSOC and FTE, which may be useful in investigating ovarian carcinogenesis and providing early detection in clinics, based on the understanding of tubal origin theory. We hypothesize that HGSOC arises from FTE, and that there should be certain differentially expressed genes between them. 
Consequently, there were two steps in the present study. Firstly, the immunophenotype and gene expression profiling among HGSOC, OSE and FTE were compared and analyzed to find evidence supporting tubal origin theory through immunohistochemistry and microarray analysis. Secondly, the candidate genes of HGSOC that were possibly associated with ovarian carcinogenesis were identified through clustering analysis and expression confirmation.

\section{Materials and methods}

Sample collection. All tissue samples were obtained from the Department of Gynecology in The Affiliated Hospital of Qingdao University between January, 2011 and May, 2012, following approval of the institutional review board from the Affiliated Hospital of Qingdao University (Qingdao, China). A total of 61 cases were used in the present study, which were assigned to three groups: 21 cases of HGSOC (range 33-69 years, median 46.6 years), 20 cases of OSE (range 47-73 years, median 49.2 years) and 20 cases of FTE (range 47-73 years, median 49.2 years) were used. The tissues samples of HGSOC were collected from patients who underwent surgical resection of ovarian tumors with written consent, while samples of FTE and OSE were obtained with written consent from patients who underwent a hysterectomy and bilateral adnexectomy owing to benign uterine disease.

Immunohistochemistry (IHC) and scoring. Immunostaining was performed to evaluate the expression of paired box gene 8 (PAX8) in the samples using Image-Pro Plus v6.0 software (Media Cybernetics, Silver Spring, MD, USA). Following fixation with $4 \%$ paraformaldehyde for $20 \mathrm{~h}$ at $23^{\circ} \mathrm{C}, 5 \mu \mathrm{m}$ paraffin-embedded sections were deparaffinized at $67^{\circ} \mathrm{C}$ for $5 \mathrm{~min}$, and rehydrated with double-distilled water. The antigens were unmasked with the heat-mediated antigen retrieval method in citrate buffer ( $\mathrm{pH}$ 6.0) (11). Incubation with the anti-PAX8 (cat. no. 10336-1-AP; Proteintech, Chicago, IL) (dilution: 1:100) primary antibody was performed at $4^{\circ} \mathrm{C}$ overnight. Specific signals were visualized by incubation with a peroxidase-conjugated secondary antibody (cat. no. ab6721; Abcam, Cambridge, MA, USA) for $60 \mathrm{~min}$ at $23^{\circ} \mathrm{C}$ followed by incubation with 3,3/-diaminobenzidine (DAB) as the chromogen at $23^{\circ} \mathrm{C}$ for $5 \mathrm{~min}$, creating a brown stain. Counterstaining with in hematoxylin for $5 \mathrm{~min}$ at $23^{\circ} \mathrm{C}$ was performed, and a coverslip was placed on the samples. Cases were scored as positive if nuclear staining was observed in $>5 \%$ of cells using a light Leica DM6000 (Leica Microsystems, GmbH, Wetzlar, Germany) at magnification, x200, as described previously (11). The scoring process was supervised by two pathologists.

mRNA expression profiling. Four samples in each of the HGSOC, FTE and OSE groups were randomly selected and prepared for microarray analysis. Total RNA was extracted from all sample tissues using the RNeasy kit (Qiagen, Inc., Valencia, CA, USA) according to the manufacturer's protocol. Microarray studies were performed by Capital Medical University Microarray Centre (Beijing, China) using Illumina humanHT-12 v4 expression BeadChip (Illumina, Inc., San Diego, CA, USA), based on the Illumina BeadStation500. Biotinylated cRNA preparation, hybridization and scanning of microarrays were performed according to the manufacturer's protocol. Triple biological replicates were used to reduce errors. Illumina Gene Expression BeadChip possesses internal control features to monitor data quality. The GenomeStudio software (version 2009.2, Illumina, Inc. San Diego, CA, USA) calculated and reported a detection $\mathrm{P}$-value, which determined whether a transcript on the array was detected. In the present study, a detection value of $\mathrm{P}<0.01$ indicated that a gene could be considered as expressed. Differentially expressed genes between HGSOC and FTE were also identified and analyzed. The output was filtered to include genes whose expression was altered at least two-fold. Gene Ontology (GO) analysis was performed to explore the cell function of differentially expressed genes using The Database for Annotation, Visualization and Integrated Discovery (DAVID) (https://david.ncifcrf.gov/tools.jsp) and the data was summarized in Results section. The microarray analysis dataset was deposited in ArrayExpress (https://www.ebi .ac.uk/arrayexpress/experiments/E-MTAB-3706/, last access date on 2 July 2015). Through the literature review in NCBI Pubmed (https://www.ncbi.nlm.nih.gov/pubmed, search term: TACTSD, ovarian cancer), the differentially expressed genes were evaluated for additional study.

Reverse transcription-quantitative polymerase chain reaction $(R T-q P C R)$. RT-qPCR was performed to confirm differential gene expression of candidate markers between HGSOC and FTE using the BIO-RAD IQ5 Real-Time PCR System (Bio-Rad Laboratories, Inc., Hercules, CA, USA). The source of total RNA was obtained from the tissues samples of HGSOC were collected from patients who underwent surgical resection of ovarian tumors with written consent, while samples of FTE were obtained with written consent from patients who underwent a hysterectomy and bilateral adnexectomy owing to benign uterine disease. The total RNA was isolated using TRIzol reagent (Invitrogen; Thermo Fisher Scientific, Inc., Waltham, MA, USA). cDNA was synthesized using $1 \mu \mathrm{g}$ total RNA, oligo (dT) 18 primer (Invitrogen; Thermo Fisher Scientific, Inc.), and Superscript ${ }^{\mathrm{TM}}$ III Reverse Transcriptase (Invitrogen; Thermo Fisher Scientific, Inc., Waltham, MA, USA). SYBR Green I was used as the fluorophore (Invitrogen; Thermo Fisher Scientific, Inc.). Synthesis was performed according to the manufacturer's protocol $\left(42^{\circ} \mathrm{C} 5 \mathrm{~min}, 95^{\circ} \mathrm{C}\right.$ for $10 \mathrm{sec}, 58^{\circ} \mathrm{C}$ for $30 \mathrm{sec}$, for 40 cycles) and quantified using the $2^{-\Delta \Delta \mathrm{Ct}}$ quantitative method (12). All the primers for TACSTD2 were designed with Primer Express software 3.0.1 (forward 5'-GCTTCCCTGTTCTGATCCTATC-3', and reverse 5'-TCTTATACTCTACCCGACCTGC-3'). $\beta$-actin was used as a reference (forward 5'-CTCCATCCTGGCCTC GCTGT-3' and reverse 5'-GCTGTCACCTTCACCGTTCC-3') (Applied Biosystems; Thermo Fisher Scientific, Waltham, MA, USA). Predicted PCR product sequences were verified using Basic local alignment search tool (BLAST; https://blast.ncbi .nlm.nih.gov/Blast.cgi) for recognition of target and non-target sequences.

Statistical analysis. Statistical analysis was performed using SPSS 17.0 (SPSS, Inc., Chicago, IL, USA). An unpaired Student's t-test was used to test for statistical significance. Data are presented as the mean \pm standard error of the 


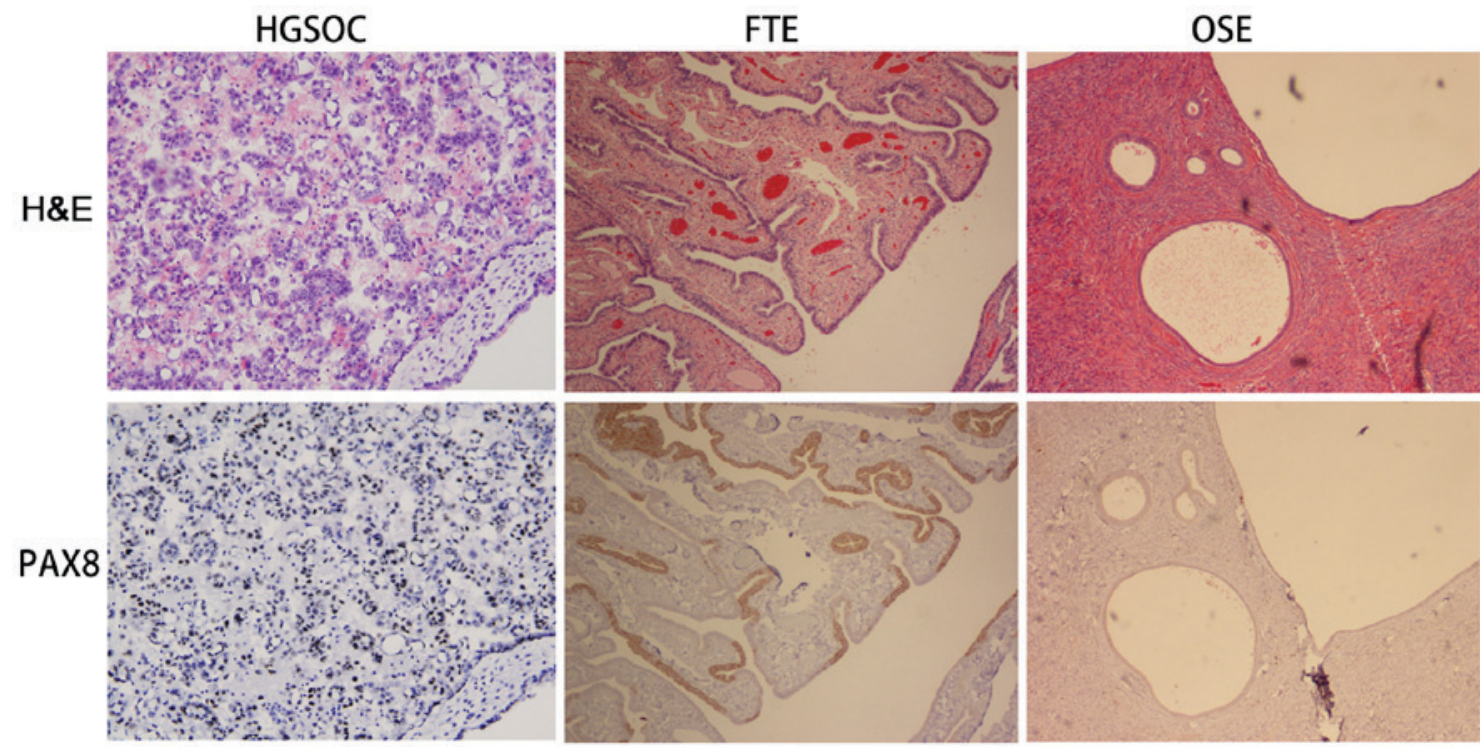

Figure 1. The different expression of PAX8 in HGSOC, FTE and OSE by H\&E (upper) and immunohistochemical (lower) staining. FTE has been considered as the tissue of Müllerian origin, while OSE links to mesothelial origin. PAX8 has been regarded as the potential marker for organs of Müllerian origin (Leica DM6000, light microscope, magnification x 200). HGSOC, high-grade serous ovarian carcinoma; FTE, fallopian tube epithelium; OSE, ovarian surface epithelium; H\&E, hematoxylin and eosin; PAX8, paired box gene 8 .

mean. $\mathrm{P}<0.05$ was considered to indicate a statistically significant difference.

\section{Results}

Immunophenotypic similarity between HGSOC and FTE. Previously, the fallopian tube was considered to be the organ of Müllerian origin, whereas OSE was associated with a mesothelial origin (8). To compare the immunophenotype of HGSOC, FTE and OSE, IHC staining was performed to confirm the expression of PAX8 (Fig. 1), which is considered to be a potential marker for organs of Müllerian origin (11). The results of the present study demonstrated that PAX8 was highly expressed in HGSOC $(19 / 21,90.4 \%)$ and FTE $(20 / 20,100 \%)$, but not in OSE $(3 / 20,14.3 \%)$. The consistency of PAX8 expression in HGSOC and FTE indicated the immunophenotypic similarity, thereby supporting a tubal origin theory.

Similarities in the gene expression profile of HGSOC and FTE. A dendrogram illustrated that HGSOC and FTE were clustered to the same branch with closer distance, when compared with OSE, indicating the presence of similarities in the gene expression profiles of HGSOC and FTE (Fig. 2A).

Differentially expressed genes between HGSOC and FTE. In total, 2,412 differentially expressed genes were identified in the microarray (absolute fold-change $>2$ ) between HGSOC and FTE, including 822 upregulated genes and 1,590 downregulated genes. The differentially expressed genes are summarized in Fig. 2B and Table I. Furthermore, Gene Ontology (GO) analysis revealed that these genes were primarily involved in the endoplasmic reticulum, extracellular region, cell fraction and structural molecule activity (Fig. 2C). The differentially expressed genes were also annotated in several Kyoto Encyclopedia of Genes and Genomes (KEGG) pathways, including focal adhesion (hsa04510), pathways in cancer (hsa05200), and the peroxisome proliferator-activated receptor (PPAR) signaling pathway (hsa03320) (https://www.ebi.ac.uk/arrayexpress/experiments/ E-MTAB-3706/).

Confirmation of the differential expression of candidate genes in HGSOC and FTE. A total of six genes differentially expressed between HGSOC and FTE were selected as candidate genes, and were confirmed via RT-qPCR using triplicate samples, including four upregulated [(S100 calcium binding protein $\mathrm{P}$ (S100P), Ras-interacting protein 1 (RASIP1), Wnt family member 5A (WNT5A), and tumor-associated calcium signal transducer 2 (TACSTD2)] and two downregulated [Dickkopf Wnt signaling pathway inhibitor 3 (DKK3 and tumor suppressor candidate 3 (TUSC3)] genes in cancer. A significant difference was detected between the expression of candidate genes in HGSOC and FTE $(\mathrm{P}<0.05)$. The data are presented in Fig. 3.

\section{Discussion}

Ovarian cancer is the most lethal gynecological cancer in women. An estimated 22,240 new cases of ovarian cancer will be diagnosed and 14,070 cancer-associated mortalities will occur in the United States in 2018, whereas rates are substantially higher in China, where a total of 52,100 incidence of ovarian cancer and 22,500 mortalities (2,3). Epithelial cancer is considered to be the most common type of ovarian cancer and is responsible for $\sim 90 \%$ of cases. Ovarian epithelial cancer consists of a heterogeneous group of histological subtypes, including serous, endometrioid, clear cell, and mucinous carcinoma. HGSOC represents the majority of cases of advanced-stage ovarian cancer and is frequently associated with a poor prognosis (7). Therefore, it is crucial to elucidate the carcinogenesis of HGSOC and identify useful tumor markers to improve treatment and prognosis. 
A

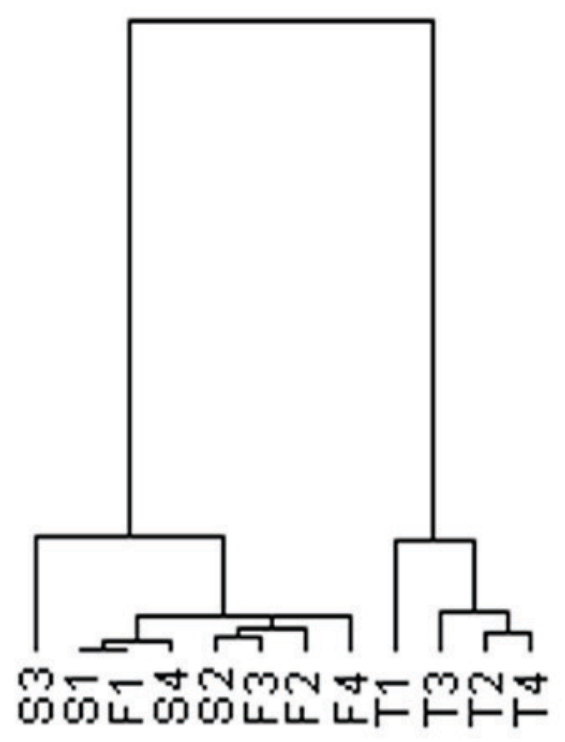

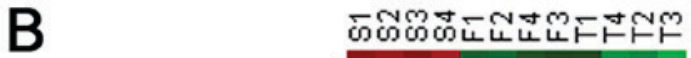

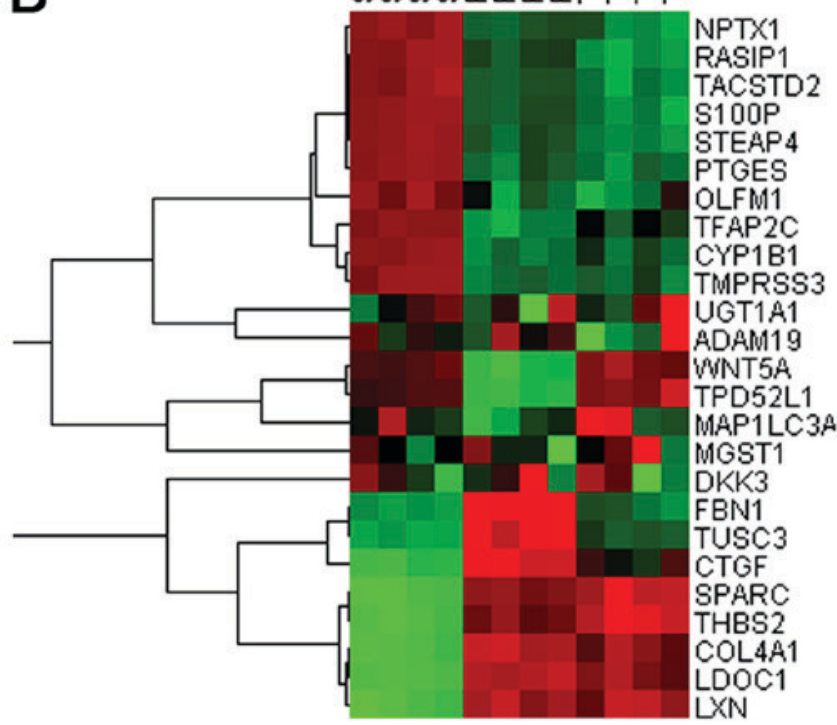

$\begin{array}{lllllll}-4.0 & -2.0 & -1.5 & 1.0 & 1.5 & 2.0 & 4.0\end{array}$

C

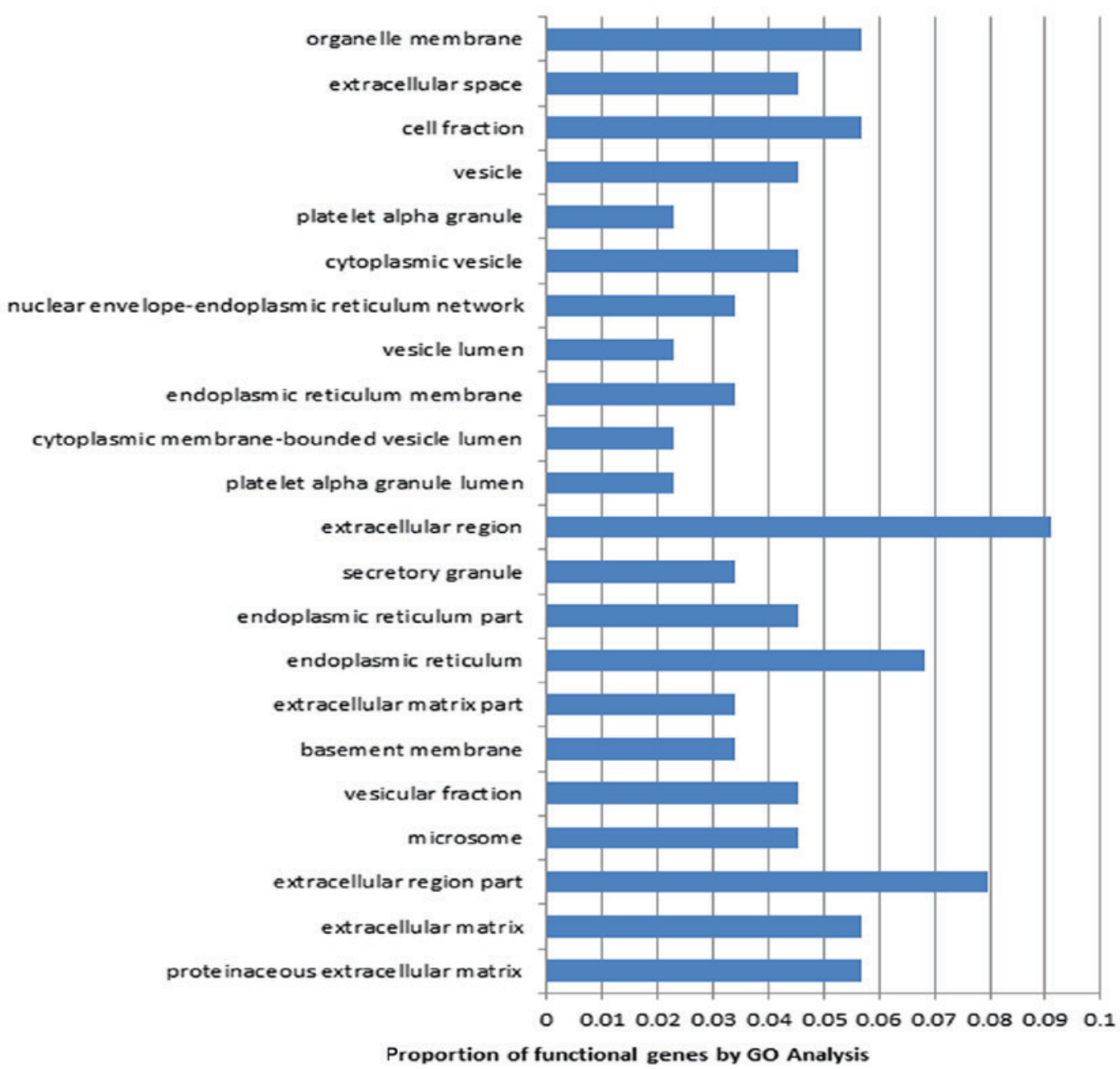

Figure 2. Gene expression profiling of HGSOC, FTE and OSE. (A) Dendrogram demonstrating that HGSOC and FTE were clustered to the same branch, compared with OSE, indicating similarities in the gene expression profiles of HGSOC and FTE. S1-S4, HGSOC samples; F1-F4, FTE samples; T1-T4, OSE samples. (B) Heatmap plot of scaled gene-expression levels through hierarchical clustering. S1-S4, HGSOC samples; F1-F4, FTE samples; T1-T4, OSE samples. (C) Gene Ontology analysis indicating that these genes, including upregulated genes and downregulated genes, were primarily involved in endoplasmic reticulum, extracellular region, cell fraction and structural molecule activity. HGSOC, high-grade serous ovarian carcinoma; FTE, fallopian tube epithelium; OSE, ovarian surface epithelium; GO, gene ontology. 
Table I. Representative differentially expressed genes between high-grade serous ovarian carcinoma and fallopian tube epithelium identified by gene expression profiling.

\section{Upregulated}

S100P

RASIP1

WNT5A

TACSTD2

PTGES

TPD52L1

MAP1LC3A

MGST1

TMPRSS3

Downregulated

DKK3

LDOC1

TUSC3

LXN
S100 calcium binding protein $\mathrm{P}$

61.3

Ras interacting protein 1

50.6

Wingless-type MMTV integration site family, member 5A

28.3

Tumor-associated calcium signal transducer 2

Prostaglandin E synthase

Tumor protein D52-like 1

19.7

Microtubule-associated protein 1 light chain 3 alpha

19.4

Microsomal glutathione S-transferase 1

16.7

Transmembrane protease, serine 3

Dickkopf WNT signaling pathway inhibitor 3

$-117.2$

Leucine zipper, down-regulated in cancer 1

Tumor suppressor candidate 3

Latexin
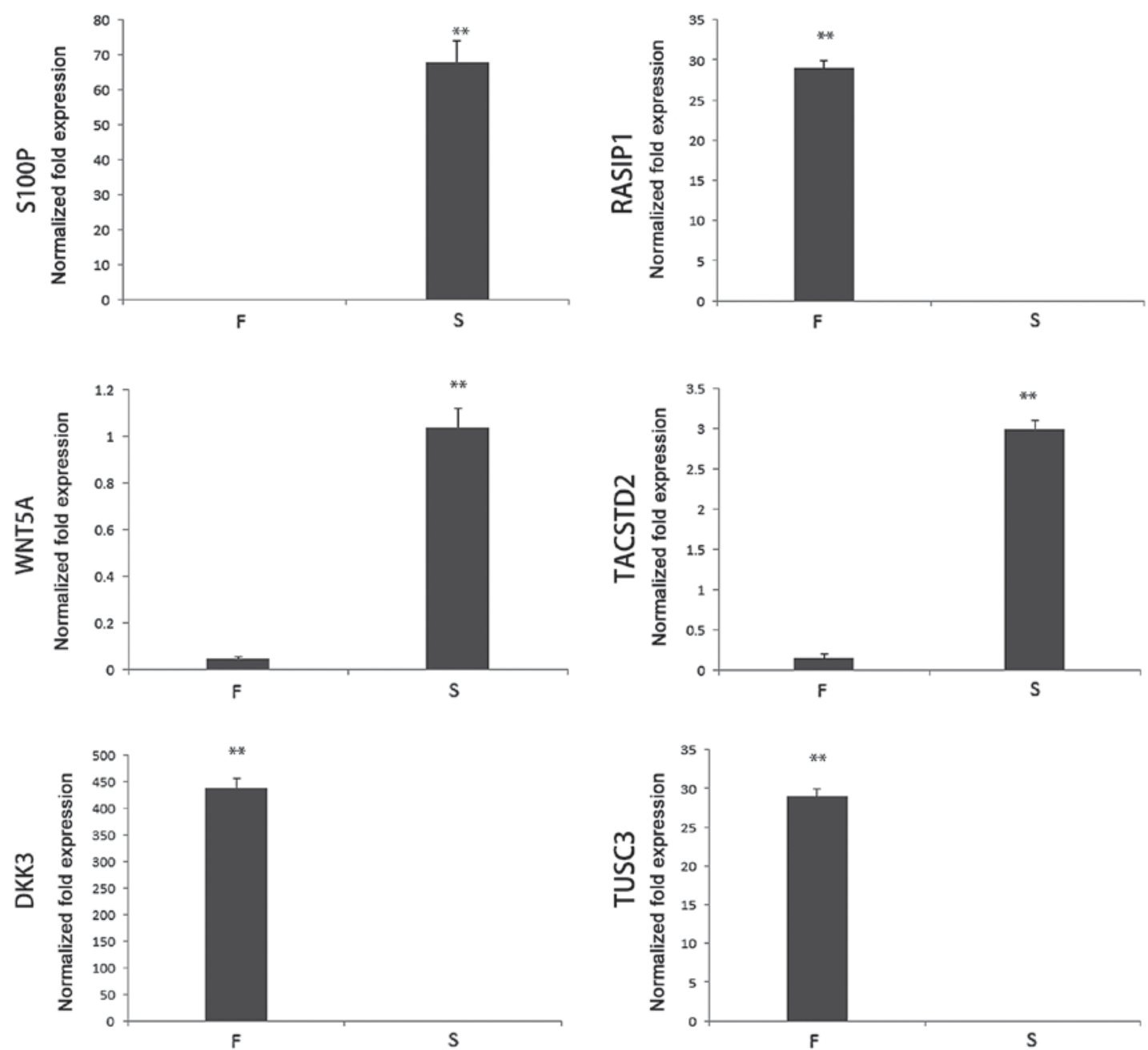

Figure 3. Differential expression of candidate genes in HGSOC and FTE $\left({ }^{* * *} \mathrm{P}<0.01\right)$. The candidate genes included genes upregulated in ovarian cancer $(\mathrm{S} 100 \mathrm{P}$, RASIP1, WNT5A, TACSTD2), as well as genes downregulated in ovarian cancer DKK3 and TUSC3. The differential expression of 6 genes in the FTE and HGSOC was confirmed by reverse transcription-quantitative polymerase chain reaction. HGSOC, high-grade serous ovarian carcinoma; FTE, fallopian tube epithelium; OSE, ovarian surface epithelium; F, FTE; S, HGSOC; S100P, S100 calcium-binding protein P; RASIP1, Ras-interacting protein 1; WNT5A, Wnt family member 5A; TACSTD2, tumor-associated calcium signal transducer 2; DKK5, Dickkopf Wnt signaling pathway inhibitor 3; TUSC3, tumor suppressor candidate 3. 
The traditional theory that ovarian cancer arises from OSE was initially proposed by Fathalla in 1971 (13). It was assumed that that the repeated overuse and repair of ovarian epithelium culminated in transformations, a concept that was further supported by epidemiological evidence revealing an increased incidence of ovarian cancer in women who had never been pregnant (14). However, the origin of ovarian cancer has been subject to controversy, and the conventional theory has been challenged: The process of transformation from OSE to ovarian cancer has never been precisely identified and defined, and ovarian cancer is more histologically similar to FTE rather than OSE (15).

A model proposing that FTE may be the origin of HGSOC has been developed. Initially, it was reported that tubal carcinoma was detected in 5 of 13 cases in prophylactic adnexectomies from women with breast cancer susceptibility protein (BRCA) mutations (BRCA positive) following a protocol of sectioning and extensively examining the fimbria (SEE-FIM); however, no ovarian carcinomas were identified, indicating that the fimbria was the most common location for early serous carcinoma in this series of BRCA-positive women (16). Subsequently, Callahan et al (17) reported that 7 consecutive cancer cases, from 123 cases of BRCA-positive women undergoing surgery for ovarian cancer risk reduction, originated in the fimbrial or ampullary region of the tube, 6 of which had an early (intraepithelial) component. This was corroborated by evidence supporting the tubal origin of ovarian cancer $(18,19)$. Clonal alterations in TP53 in benign tubal epithelium, which are referred to as p53 signatures, and generic secretory cell outgrowth (SCOUT) in the FTE associated with altered PAX2 expression has established a foundation for a serous cancer precursor in the fimbria (10). Accordingly, the Society of Gynecologic Oncology recommendations for the prevention of ovarian cancer indicate the importance of the FTEs as a potential source of HGSOC (20). Furthermore, in parallel with the implementation of the new International Federation of Gynecology and Obstetrics (FIGO) staging classification (21), the revised World Health Organization (WHO) classification eliminates the previous focus on the mesothelial origin of ovarian cancer, and features a discussion of tubal carcinogenesis of hereditary and other types of high-grade serous carcinomas (6).

Evidence has been provided for the theoretical tubal origin of HGSOC, and gynecologists recommend a preventative bilateral salpingectomy for women with a high-risk of ovarian cancer (20). However, at the time of writing, the tubal origin of HGSOC has not been demonstrated completely or become the established clinical guideline based on FIGO and WHO files $(6,20-22)$. The present study aimed to assess the possibility and feasibility of identifying the genes differentially expressed between HGSOC and FTE, which may be useful in investigating ovarian carcinogenesis. The candidate genes identified in the present study are relatively novel, including 4 upregulated (S100P, RASIP1, WNT5A and TACSTD2), and 2 downregulated (DKK3 and TUSC3) genes in cancer. In the present study, PAX8 was used for immunostaining as a recognized marker for FTE and HGSOC, hence its selection for the immunophenotypic comparison of FTE, HGSOC and OSE. The similarity establishment of FTE and HGSOC was the first step in the present study. Gene expression profile similarity was then confirmed and the differentially expressed genes were identified. There were a total of 2,412 differentially expressed genes analyzed recognized in the microarray of the present study. Factors, including fold-change, association with tumor origin and development, and current research status were taken into consideration when deciding which genes to study further. Upregulated and downregulated genes in cancer following the order of fold-change were listed and the published articles of the top 100 genes in each list were reviewed to estimate their research status and tumor association. Through the literature review in NCBI Pubmed, S100P, RASIP1, WNT5A, TACSTD2, DKK3 and TUSC3, which are closely associated with tumor development, were chosen as the candidate markers in the present study.

Accumulating evidence has demonstrated that the aforementioned candidate genes are involved in the tumorigenesis and progression of multiple cancer types, including ovarian cancer. For example, high expression of S100P is associated with unfavorable prognosis and tumor progression in patients with epithelial ovarian cancer $(23,24)$. Post et al $(25)$ reported that RASIP1 mediates Rap1 regulation of Rho in endothelial barrier function through ArhGAP29, which may serve an important role in the development of tumors (25). Studies have demonstrated that WNT5A exerts immunomodulatory activity and influences viability, migration, adhesion, colony formation and the expression of $\mathrm{E}$ - and $\mathrm{N}$-cadherin in the human ovarian cancer SKOV-3 cell line $(26,27)$. TACSTD2 is an intracellular calcium signal transducer that is differentially expressed in a number of cancer types (28). DKK3 may serve a key role in the inhibition of cancer cell proliferation by regulating Wnt signaling (29). Expression of TUSC3 prevents the epithelial-to-mesenchymal transition and inhibits tumor growth by modulating the endoplasmic reticulum stress response in ovarian cancer cells (30). Further study of these candidate genes is necessary to investigate the molecular mechanism of cancer development.

To conclude, in the present study, a greater similarity of immunophenotype and gene expression profile was observed between HGSOC and FTE when compared with OSE, and a total of 6 candidate genes that are potentially associated with the carcinogenesis of HGSOC were identified. It is evident that the present study is relatively superficial, and merely offers supportive evidence for tubal origin theory and potential gene markers for future study. However, it is possible that any novel knowledge regarding the tubal origin of ovarian cancer may open novel pathways in basic research and clinical studies.

\section{Acknowledgements}

Not applicable.

\section{Funding}

The present study was supported by National Natural Science Fund (grant nos. 81402157 and 81502310) and the Doctoral Fund of The Affiliated Hospital of Qingdao University (grant no. 2075). The funders had no role in study design, data collection and analysis, decision to publish, or preparation of the manuscript. 


\section{Availability of data and materials}

The microarray analysis dataset was deposited in ArrayExpress (accession no. E-MTAB-3706, https://www.ebi.ac.uk/arrayexpress/experiments/E-MTAB-3706/).

\section{Author contributions}

XL conceived and designed the experiments, GR and ZJ performed the experiments: GR and WZ analyzed the data, SF collected the samples and cases and XL wrote the paper.

\section{Ethics approval and consent to participate}

The study was approved by the Institutional Review Board and Human Ethics Committee of Affiliated Hospital of Qingdao University. Written informed consent for using the samples for research purposes was obtained from all patients prior to surgical resection.

\section{Consent for publication}

The authors declare that the patient, parent, guardian or next of kin (in case of deceased patients) provided written informed consent for the publication of any associated data and accompanying images.

\section{Competing interests}

The authors declare that they have no competing interests.

\section{References}

1. Kujawa KA and Lisowska KM: Ovarian cancer-from biology to clinic. Postepy Hig Med Dosw (Online) 69: 1275-1290, 2015 (Article in Polish).

2. Siegel RL, Miller KD and Jemal A: Cancer statistics, 2018. CA Cancer J Clin 68: 7-30, 2018

3. Chen W, Zheng R, Baade PD, Zhang S, Zeng H, Bray F, Jemal A, $\mathrm{Yu}$ XQ and He J: Cancer statistics in China, 2015. CA Cancer J Clin 66: 115-132, 2016.

4. Eng KH, Hanlon BM, Bradley WH and Szender JB: Prognostic factors modifying the treatment-free interval in recurrent ovarian cancer. Gynecol Oncol 139: 228-235, 2015.

5. Ramalingam P: Morphologic, immunophenotypic, and molecular features of epithelial ovarian cancer. Oncology (Williston Park) 30: 166-176, 2016.

6. Meinhold-Heerlein I, Fotopoulou C, Harter P, Kurzeder C, Mustea A, Wimberger P, Hauptmann S and Sehouli J: The new WHO classification of ovarian, fallopian tube, and primary peritoneal cancer and its clinical implications. Arch Gynecol Obstet 293: 695-700, 2016

7. Kurman RJ: Origin and molecular pathogenesis of ovarian high-grade serous carcinoma. Ann Oncol 24(Suppl 10): S16-S21, 2013.

8. Perets $\mathrm{R}$ and Drapkin R: It's totally Tubular...Riding the new wave of ovarian cancer research. Cancer Res 76: 10-17, 2016.

9. Ning G, Bijron JG, Yamamoto Y, Wang X, Howitt BE, Herfs M, Yang E, Hong Y, Cornille M, Wu L, et al: The PAX2-null immunophenotype defines multiple lineages with common expression signatures in benign and neoplastic oviductal epithelium. J Pathol 234: 478-487, 2014.

10. Mehra K, Mehrad M, Ning G, Drapkin R, McKeon FD, Xian W and Crum CP: STICS, SCOUTs and p53 signatures a new language for pelvic serous carcinogenesis. Front Biosc (Elite Ed) 3: 625-634, 2011.
11. Xiang L, Zheng W and Kong B: Detection of PAX8 and p53 is beneficial in recognizing metastatic carcinomas in pelvic washings, especially in cases with suspicious cytology. Gynecol Oncol 127: 595-600, 2012.

12. Livak KJ and Schmittgen TD: Analysis of relative gene expression data using real-time quantitative PCR and the 2 (-Dalta Dalta C(T)) method. Methods 25: 402-408, 2001.

13. Fathalla MF: Incessant ovulation-a factor in ovarian neoplasia? Lancet 2: 163, 1971.

14. Fraumeni JF Jr, Lloyd JW, Smith EM and Wagoner JK: Cancer mortality among nuns: Role of marital status in etiology of neoplastic disease in women. J Natl Cancer Inst 42: 455-468, 1969.

15. Mhawech-Fauceglia P, Wang D, Samrao D, Godoy H, Ough F Liu S, Pejovic T and Lele S: Pair Box 8 (PAX8) protein expression in high grade, late stage (stages III and IV) ovarian serous carcinoma. Gynecol Oncol 127: 198-201, 2012.

16. Medeiros F, Muto MG, Lee Y, Elvin JA, Callahan MJ, Feltmate C, Garber JE, Cramer DW and Crum CP: The tubal fimbria is a preferred site for early adenocarcinoma in women with familial ovarian cancer syndrome. Am J Surg Pathol 30: 230-236, 2006.

17. Callahan MJ, Crum CP, Medeiros F, Kindelberger DW, Elvin JA, Garber JE, Feltmate CM, Berkowitz RS and Muto MG: Primary fallopian tube malignancies in BRCA-positive women undergoing surgery for ovarian cancer risk reduction. J Clin Oncol 25: 3985-3990, 2007.

18. Seidman JD: Serous tubal intraepithelial carcinoma localizes to the tubal-peritoneal junction: A pivotal clue to the site of origin of extrauterine high-grade serous carcinoma (ovarian cancer). Int J Gynecol Pathol 34: 112-120, 2015.

19. Erickson BK, Conner MG and Landen CN Jr: The role of the fallopian tube in the origin of ovarian cancer. Am J Obstet Gynecol 209: 409-414, 2013.

20. Walker JL, Powell CB, Chen LM, Carter J, Bae Jump VL, Parker LP, Borowsky ME and Gibb RK: Society of gynecologic oncology recommendations for the prevention of ovarian cancer. Cancer 121: 2108-2120, 2015.

21. Duska LR and Kohn EC: The new classifications of ovarian, fallopian tube, and primary peritoneal cancer and their clinical implications. Ann Oncol 28(Suppl 8): 2017.

22. Long Roche KC, Abu-Rustum NR, Nourmoussavi M and Zivanovic O: Risk-reducing salpingectomy: Let us be opportunistic. Cancer 123: 1714-1720, 2017.

23. Wang X, Tian T, Li X, Zhao M, Lou Y, Qian J, Liu Z, Chen H and Cui Z: High expression of S100P is associated with unfavorable prognosis and tumor progression in patients with epithelial ovarian cancer. Am J Cancer Res 5: 2409-2421, 2015.

24. Surowiak P, Maciejczyk A, Materna V, Drag-Zalesińska M, Wojnar A, Pudelko M, Kedzia W,Spaczyński M, Dietel M, Zabel M and Lage H: Unfavourable prognostic significance of S100P expression in ovarian cancers. Histopathology 51: 125-128, 2007.

25. Post A, Pannekoek WJ, Ross SH, Verlaan I, Brouwer PM and Bos JL: Rasip1 mediates Rap1 regulation of Rho in endothelial barrier function through ArhGAP29. Proc Natl Acad Sci USA 110: 11427-11432, 2013.

26. Arabzadeh S, Hossein G and Zarnani AH: Wnt5A exerts immunomodulatory activity in the human ovarian cancer cell line SKOV-3. Cell Biol Int 40: 177-187, 2016.

27. Jannesari-Ladani F, Hossein G, Monhasery N, Shahoei SH and Izadi Mood N: Wnt5a influences viability, migration, adhesion, colony formation, E- and N-cadherin expression of human ovarian cancer cell line SKOV-3. Folia Biol (Praha) 60: 57-67, 2014.

28. Shvartsur A and Bonavida B: Trop 2 and its overexpression in cancers: Regulation and clinical/therapeutic implications. Genes Cancer 6: 84-105, 2015.

29. Mohammadpour H, Pourfathollah AA, Nikougoftar Zarif M and Khalili S: Key role of Dkk3 protein in inhibition of cancer cell proliferation: An in silico identification. J Theor Biol 393: 98-104, 2016.

30. Kratochvílová K, Horak P, Ešner M, Souček K, Pils D, Anees M, Tomasich E, Dráfi F, Jurtíková V, Hampl A, et al: Tumor suppressor candidate 3 (TUSC3) prevents the epithelial-to-mesenchymal transition and inhibits tumor growth by modulating the endoplasmic reticulum stress response in ovarian cancer cells. Int J Cancer 137: 1330-1340, 2015.

This work is licensed under a Creative Commons Attribution-NonCommercial-NoDerivatives 4.0 International (CC BY-NC-ND 4.0) License. 